\title{
Low-dose three-dimensional hard x-ray imaging of bacterial cells
}

\author{
Matthias Bartels ${ }^{1 *}$, Marius Priebe ${ }^{1}$, Robin N Wilke ${ }^{1}$, Sven P Krüger ${ }^{1}$, Klaus Giewekemeyer ${ }^{1}$, \\ Sebastian Kalbfleisch ${ }^{1}$, Christian Olendrowitz ${ }^{1}$, Michael Sprung ${ }^{2}$ and Tim Salditt ${ }^{{ }^{*}}$
}

\begin{abstract}
We have imaged the three-dimensional density distribution of unstained and unsliced, freeze-dried cells of the gram-positive bacterium Deinococcus radiodurans by tomographic x-ray propagation microscopy, i.e. projection tomography with phase contrast formation by free space propagation. The work extends previous $x$-ray imaging of biological cells in the simple in-line holography geometry to full three-dimensional reconstruction, based on a fast iterative phase reconstruction algorithm which circumvents the usual twin-image problem. The sample is illuminated by the highly curved wave fronts emitted from a virtual quasi-point source with $10 \mathrm{~nm}$ cross section, realized by two crossed $x$-ray waveguides. The experimental scheme allows for a particularly dose efficient determination of the 3D density distribution in the cellular structure.
\end{abstract}

Keywords: X-ray optics, X-ray waveguides, Synchrotron radiation instrumentation, Phase contrast, Phase retrieval, Tomography

\section{Background}

Optical nanoscopy based on visible light fluorescence is becoming an important tool for three-dimensional imaging of biological cells at the nanoscale. However, not all biological problems can be addressed based on the distribution of fluorescence markers. In many instances additional and complementary contrast mechanisms are needed. The mass density distribution within native unstained and unsliced biological cells and tissues is such a case in point. To this end, coherent $\mathrm{x}$-ray imaging and tomography offers a unique potential for quantitative three-dimensional (3D) density determination at scalable resolution (Cloetens et al. 2006; Huang et al. 2009; Lima et al. 2009; Nishino et al. 2009; Shapiro et al. 2005; Song et al. 2008). The distribution of density contrast in the sample is obtained from quantitative phase reconstruction schemes ((Nugent 2010; Paganin 2006), and ref. therein), along with the reconstructed volumes, shapes and topologies. The density based contrast mechanism

\footnotetext{
*Correspondence: mbartel@gwdg.de; tsaldit@gwdg.de

1 Institut für Röntgenphysik, Georg-August-Universität Göttingen,

Friedrich-Hund-Platz 1, 37077 Göttingen, Germany

Full list of author information is available at the end of the article
}

equips biophysical research with a non-destructive 'ultracentrifuge' at the organelle and supra-molecular level. In other words, quantitative mass density measurements and density based discrimination, which is important in many biological applications, but typically associated with destructive separation of components, can be performed non-destructively. Important problems associated with sub-cellular architecture, as for example DNA compactification can thus be addressed. However, for biological samples the applied dose is a crucial parameter in view of structural changes and radiation damage during the imaging process, inhibiting live cell imaging and creating a need for fixation. Moreover, radiation damage of samples is considered to be the ultimate limitation in $\mathrm{x}$ ray microscopy, if resolution due to the characteristics of sources, optical components, reconstruction algorithms and detection is successfully scaled down (Howells et al. 2009). Therefore, minimizing the dose for a given image resolution and contrast is a primary challenge for $\mathrm{x}$-ray optics.

Here we present a 3D tomographic reconstruction of unstained freeze-dried cells of the gram-positive bacterium Deinococcus radiodurans using hard x-rays (13.8 keV photon energy). The total dose applied during

\section{Springer}

(c) 2012 Bartels et al: licensee Springer. This is an Open Access article distributed under the terms of the Creative Commons Attribution License (http://creativecommons.org/licenses/by/2.0), which permits unrestricted use, distribution, and reproduction in any medium, provided the original work is properly cited. 
the tomographic scan was about $1.6 \cdot 10^{5}$ Gray ([GY=J/kg]), corresponding to $1.9 \cdot 10^{3}$ Gy per projection, which is several orders of magnitude below the values reported in previous $x$-ray coherent diffractive imaging (CDI) studies of $D$. radiodurans (Lima et al. 2009; Giewekemeyer et al. 2010; Wilke et al. 2012). Despite the low dose, phase reconstruction based on recorded holographic intensities was not hampered by noise, primarily due to two reasons: (i) the use of a highly coherent beam with curved wavefronts (Salditt et al. 2011), emitted by a quasi-point source with $10 \mathrm{~nm}$ cross section, realized by a $2 \mathrm{D}$ x-ray waveguide system (Krüger et al. 2010, 2012), and (ii) a robust and quickly converging iterative reconstruction scheme which takes photon noise effects into account quantitatively (Giewekemeyer et al. 2011). The electron density distribution and an effective mass density distribution of the cellular structure was extracted quantitatively from the reconstructed 3D phase information. Density contrast may help to understand the bacterium's extraordinary resistance to high doses of ionizing radiation based on the structural arrangement of its nucleoid (Eltsov and Dubochet 2006a, 2006b; Minskey et al. 2006). Coherent $\mathrm{x}$-ray imaging was previously used to derive the projected electron density of $D$. radiodurans (Giewekemeyer et al. 2010), providing thus a contrast mechanism complementary to electron microscopy studies (Eltsov and Dubochet 2005; Levin-Zaidman et al. 2003). The data was recorded by scanning a micron-sized $\mathrm{x}$-ray beam over the sample, followed by reconstruction of the farfield diffraction patterns, yielding the super-resolution real space projection image. The reconstruction algorithm used was based on the a priori knowledge of partial overlap between adjacent images, reducing the number of unknown variables, an approach denoted by ptychography (Dierolf et al. (2010)). In order to obtain the locally resolved electron and mass density rather than just projected values, we here extend this work from $2 \mathrm{D}$ to $3 \mathrm{D}$.

However, instead of ptychographic tomography which involves scanning two translations and one rotation with correspondingly large overhead time (Dierolf et al. 2010; Wilke et al. 2012), in this work we use $\mathrm{x}$-ray propagation microscopy, i.e. projection imaging with contrast formation by free space propagation (Cloetens et al. 1999; Wilkins et al. 1996). In our view, replacing the (Fraunhofer) far-field recording with a magnified (Fresnel) nearfield setting (Giewekemeyer et al. 2011, Mokso et al. 2007) has the following advantages: (i) The near-field pattern directly represents location and shape of the object, so that object support and position in the beam can be readily located, giving useful $a$ priori knowledge. (ii) Current detector technology is better exploited due to homogenous signal level. Furthermore, (iii) a high dose efficiency is gained by interference of the weak diffracted wave with the much stronger primary wave as demonstrated experimentally in this work. Finally, (iv) in view of 3D imaging, a full field approach based on scanning only one degree of freedom (rotation axis) produces less overhead time in data acquisition and imposes less restrictive requirements on mechanical accuracy and longterm vibrational stability. However, shaping a highly coherent beam with curved wavefronts is a major challenge in propagation imaging. By using $\mathrm{x}$-ray waveguides the well-known problems of empty beam correction and geometrical distortions associated with hard x-ray focusing optics (Mokso et al. 2007) can be solved (Giewekemeyer et al. 2011).

\section{Methods}

Figure 1 shows a schematic of the experiment performed with the Göttingen Instrument for Nano Imaging with $X$-rays (GINIX) installed at the coherence beamline P10 of the new storage ring PETRA III at Hasylab, DESY (Kalbfleisch et al. 2011; Salditt et al. 2011). The undulator beam was monochromatized by a $\mathrm{Si}(111)$ double crystal to $13.8 \mathrm{keV}$ and then focused by two Kirkpatrick-Baez (KB) mirrors polished to fixed elliptical shape, yielding a focus size of $D_{\text {horz }}=370 \mathrm{~nm}$ and $D_{\text {vert }}=200 \mathrm{~nm}$ full width at half maximum (FWHM), as measured by scanning $\mathrm{x}$-ray waveguides horizontally and vertically through the beam. The total flux was $2.4 \times 10^{11}$ counts per second at $70 \mathrm{~mA}$ ring current, as measured by a pixel detector (Pilatus, Dectris) positioned at $5.29 \mathrm{~m}$ in the widened far-field of the KB beam. The waveguide (WG) system was positioned in the focal plane of the KB mirror, using a miniaturized fully motorized goniometer with optical encoders (Attocube). Alignment of the waveguide as well as the sample mounted on a dedicated tomography stage was facilitated by use of two on-axis optical microscopes, one directed downstream and one upstream with the beam, see Additional file 1 for details. Two different single photon counting pixel detectors, with $172 \mu \mathrm{m}$ (Pilatus, Dectris) and $55 \mu \mathrm{m}$ (Maxipix) pixel size were used. The waveguide system consisted of two crossed planar waveguide slices, each with a transmission optimized sputtered thin film sequence $\mathrm{Ge} / \mathrm{Mo} / \mathrm{C} / \mathrm{Mo} / \mathrm{Ge}$, with $35 \mathrm{~nm}$ amorphous $C$ as the guiding layer (Krüger et al. 2010, 2012; Salditt et al. 2011). Note that due to grazing angles, the guiding layer width of $35 \mathrm{~nm}$ corresponds to only two propagating modes in the waveguide. Absorption of radiative modes in the cladding then results in a strong damping of all radiation except of the two fundamental modes. Finally, the interference of the modes can lead to a an effective beam width which is substantially smaller than the guiding layer thickness (Krüger et al. 2012). The small exit beam width of the waveguide beam is evidenced by the large divergence angle of its far-field intensity distribution, measured with 

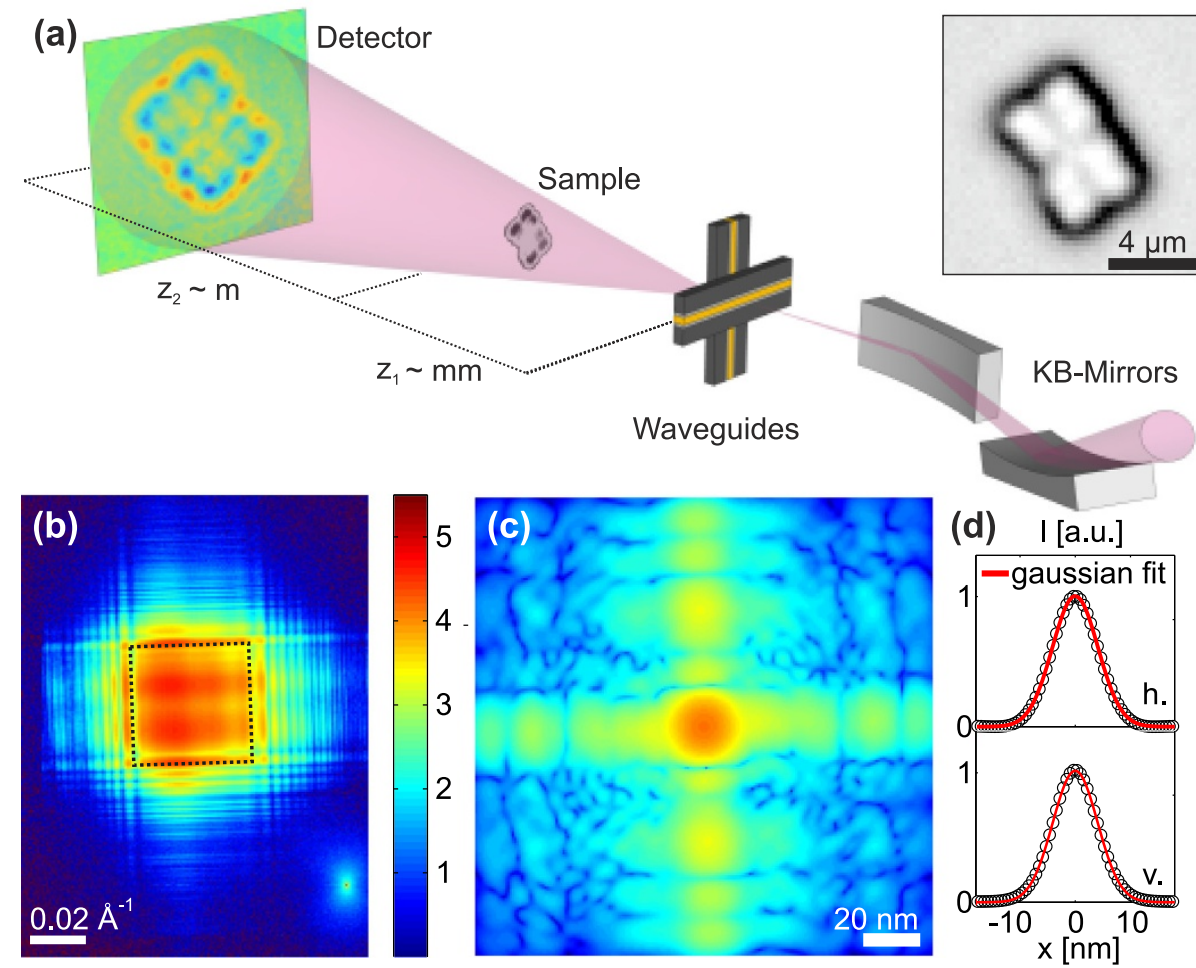

Figure 1 Experimental setup. (a) A monochromatic $x$-ray beam is focused by two KB mirrors onto the system of two crossed planar waveguides (WG) with a layered guiding core structure for optimized transmission. The sample (for an optical mircograph of the cells, see inset) is placed at a distance $z_{1}$ behind the WG exit. The area pixel detector is placed at a distance $z_{2} \gg z_{1}$ away from the sample to collect the diffraction pattern. (b) Logarithmic far-field intensity distribution of a $15 \mathrm{keV}$ beam exiting the waveguide (scale bar $0.02 \AA^{-1}, 100$ second dwell time), measured with a pixel detector (Pilatus) at a distance of $5.29 \mathrm{~m}$ behind the focus. The small focal width of the waveguide beam is evidenced by its large divergence angle, the relatively homogenous intensity distribution well suited for imaging (dashed rectangle) has a divergence angle of 5 mrad. (c) The

near-field intensity distribution in the effective focal plane is obtained by inverting the diffraction pattern with the error reduction algorithm, shown in logarithmic scale, scale bar $20 \mathrm{~nm}$. (d) Gaussian fits of the central peak along the horizontal and vertical direction give a width of $10 \mathrm{~nm} \times 9.8 \mathrm{~nm}$ (FWHM), respectively.

a pixel detector (Pilatus) at a distance of $5.29 \mathrm{~m}$ behind the WG (data shown in Figure 1(b), recorded at $15 \mathrm{keV}$ photon energy). The relatively homogenous part of the waveguide far-field intensity distribution (dashed rectangle in Figure 1(b)) is well suited for imaging and has a divergence angle of $5 \mathrm{mrad}$. The near-field intensity distribution in the effective focal plane is obtained by inverting the diffraction pattern using the error reduction algorithm (Krüger et al. 2010, 2012 (see Figure 1(c)). Gaussian fits of the central peak along the horizontal and vertical direction give a width of $10 \mathrm{~nm} \times 9.8 \mathrm{~nm}$ (FWHM), respectively (see Figure 1(d)). Cells of the Deinococcus radiodurans wild-type strain were cultivated from freeze-dried cultures (DSM No. 20539 by the German Collection of Microorganisms and Cell Cultures), suspended on $\mathrm{Si}_{3} \mathrm{~N}_{4}$-foils (Silson) with $1 \mu \mathrm{m}$ thickness and $5 \times 5 \mathrm{~mm}^{2}$ lateral dimensions, shock frozen by cryogenic fixation in ethane (cryo-plunging), and subsequently freeze dried, see Additional file 1 for details. To record projection propagation images in full field mode the sample (for an optical micrograph of the cells, see Figure 1(a)) was placed at a (defocus) distance $z_{1}=8$ $\mathrm{mm}$ from the WG exit plane, where the divergent WG beam has broadened to a field of view (FOV) of $40 \times$ $40 \mu \mathrm{m}^{2}$, as calculated from the measured far-field divergence angle of $5 \mathrm{mrad}$. As shown previously, the imaging experiment can then be described in a well-known equivalent parallel-beam geometry (Mayo et al. 2002; Fuhse et al. 2006) with a demagnified (effective) detector pixel size of $\Delta_{D} / M$ and a (de)magnification factor of $M=\left(z_{1}+z_{2}\right) / z_{1}$ as well as an effective sample-detector distance $z_{\text {eff }}=z_{1} z_{2} /\left(z_{1}+z_{2}\right)=z_{2} / M$. Note that here $z_{2} \gg z_{1}$, so that $z_{\text {eff }} \simeq z_{1}=8 \mathrm{~mm}$ and $M \simeq z_{2} / z_{1}=660$, resulting in an effective detector pixel size of $\simeq 83 \mathrm{~nm}$. For 3D imaging, 83 projection images $I_{\phi}$ were collected over 162 degrees with a total exposure time of $10 \mathrm{~min}$ utes for each angle $\phi$, distributed over $N=15$ detector accumulations, which were subsequently corrected for lateral drift by cross-correlation methods with sub-pixel accuracy (Guizar-Sicairos et al. 2008). The total fluence 
for each projection was $\simeq 4.38 \cdot 10^{6}$ photons $/ \mu \mathrm{m}^{2}$, corresponding to a dose of $\simeq 1.9 \cdot 10^{3} \mathrm{~Gy}$, based on calculations presented in (Howells et al. 2009). Next, the normalized intensity distribution was calculated as $\overline{I_{\phi}}(x, y)=I_{\phi} / I_{0}$ by division with the empty beam intensity distribution $I_{0}(x, y)$, followed by a filtering of residual low frequency variations, see Additional file 1 for details on image processing and filtering. Based on the projection approximation, the measured signal at the detector normalized by the empty beam intensity distribution, described in the equivalent parallel-beam geometry is then given as

$$
\overline{I_{\phi}}(x, y)=\left|D_{z_{\mathrm{eff}}}[\chi(x, y)]\right|^{2},
$$

where $D_{z_{\mathrm{eff}}}$ denotes the two-dimensional Fresnel propagator over the distance $z_{\text {eff }}$ along the optical axis and $\chi(x, y)$ the sample transmission function (Giewekemeyer et al. 2011). Backpropagation of Eq. (1) leads to a single-step holographic reconstruction of the normalized intensity $\overline{I_{\phi}}(x, y)$, which is however intrinsically spoiled by the socalled twin-image. As presented previously in Giewekemeyer et al. (2011), a significant improvement of the reconstruction can be achieved by application of a modified version of the Hybrid-Input-Output (HIO) algorithm (Paganin (2006)) well suited for pure phase objects. Note that the HIO algorithm is a classical iterative algorithm, which cycles between reciprocal space and real space. In reciprocal space, the measured information is fed in, and in real space, additional 'real space' constrains are applied, which in many cases leads to convergence towards the solution which satisfied both constraints. Most common a priori knowledge in real space is based on support information, i.e. in which region of the illuminated area the sample is positioned and/or amplitude constraint, i.e. knowledge about the amplitude of the object, for example when absorption can be neglected. According to the
HIO version published in Giewekemeyer et al. (2011), the real space upgrade consists of two constraints, one for the amplitude, and one for the phase of the wave, as specified by the following two equations. The update of the current amplitude $\left|\chi_{n}\right|$ is given by

$$
\left|\chi_{n+1}\right|=\left|\chi_{n}\right|-\beta \cdot\left(\left|\chi_{n}^{\prime}\right|-1\right),
$$

as proposed in Gurevey (2003). Secondly, a phase constraint is included in the iteration, namely,

$$
\begin{aligned}
& \varphi\left(\chi_{n+1}(x, y)\right) \\
& =\left\{\begin{array}{r}
\varphi\left(\chi_{n}(x, y)\right)-\gamma \cdot \varphi\left(\chi^{\prime}{ }_{n}(x, y)\right) \forall(x, y) \notin S \\
\min \left\{\varphi\left(\chi^{\prime}{ }_{n}(x, y)\right), 0\right\} \forall(x, y) \in S .
\end{array}\right.
\end{aligned}
$$

$\left|\chi_{n}^{\prime}{ }_{n}\right|$ denotes the amplitude of the $n$-th iterate after application of the detection plane constraint, i.e. $\chi_{n}^{\prime}:=$ $P_{M}\left(\chi_{n}\right)$ with $P_{M}\left(\chi_{n}\right)=D_{-z_{\mathrm{eff}}}\left[\sqrt{\bar{I}} \cdot \exp \left(i \varphi\left(\tilde{\chi}_{n}\right)\right)\right]$ denoting the modulus replacement operation in the detection plane and $\varphi(z):=\arg (z)$ for any complex number $z \in \mathbb{C}$. The amplitude constraint (2) slowly pushes $\left|\chi_{n}\right|$ towards 1 and the phase constraint (3) causes a gentle decrease of the phase to a constant $C$ ( $C=0$ was chosen here) in the area, where no object is located. The phase inside the support area $S \subset \mathbb{R}^{2}$, however, is left untouched, as long as it is not larger than $C$, allowing for phase changes $\Delta \varphi(x, y)$ in one direction only, as expected for objects with $|\Delta \varphi(x, y)|<$ $\pi$. The speed of convergence is determined by the feedback parameters $\gamma \in[0,1]$ and $\beta \in[0,1]$. For tomographic datasets it is crucial to determine the accurate support area $S$ automatically for each projection, which is achieved by a thresholding approach, as explained in Additional file 1.

Before application to a biological sample, suitable test structures can be used to control the contrast transfer of the setup at the relevant resolution range. Figure 2 shows the example of a lithographic pattern consisting of $50 \mathrm{~nm}$
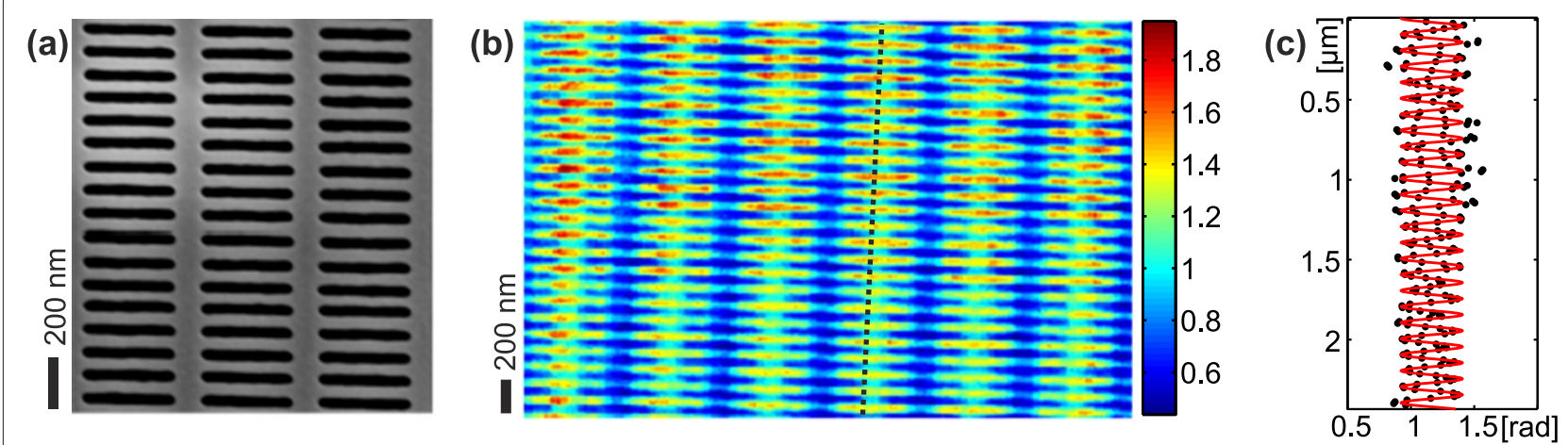

Figure 2 Test structure. (a) Scanning electron micrograph of a $500 \mathrm{~nm}$ thick tantalum test structure with $50 \mathrm{~nm}$ lines and spaces. (b) Hologram of the test structure obtained from the normalized intensity distribution recorded during 1 second total illumination time. Effective pixel sizes are 16 $\mathrm{nm}$ in horizontal and $11 \mathrm{~nm}$ in vertical direction, the field of view is $3.4 \mu \mathrm{m}(\mathrm{h}) \times 2.2 \mu \mathrm{m}$ (v). (c) Profile along the black line in (b) together with a sinusoidal fit yielding a perdiodicity of $100.7 \mathrm{~nm}$, well in agreement with the $50 \mathrm{~nm}$ feature size. 
lines and spaces in a $500 \mathrm{~nm}$ thick tantalum layer (NTTAT, Japan, model \# ATN/XRESO-50HC), see (a) for a SEM micrograph. The measured hologram (b), after correction by the empty beam, corresponds to a total field of view of $3.4 \mu \mathrm{m}(\mathrm{h}$.) $\times 2.2 \mu \mathrm{m}(\mathrm{v}$.). Analysis of line cuts as shown in (c) shows that sufficient contrast of $\Delta I / I \simeq 0.2$ is achieved. For the data on the test structure, we have used a waveguide system with $60 \mathrm{~nm}$ amorphous $C$ as the guiding layer resulting in a total flux of $5 \times 10^{8} \mathrm{cps}$ exiting the waveguide system at $80 \mathrm{~mA}$ ring current. The test structure was positioned at $z_{1}=1.063 \mathrm{~mm}$. Due to the sequential arrangement of the crossed waveguide system the distances from both individual planar waveguide slices to the sample differ by $450 \mu \mathrm{m}$, which is the thickness of the second waveguide slice. With the detector (Maxipix) placed at $z_{2}=5.36 \mathrm{~m}$ the effective pixel side lengths are $\simeq 16 \mathrm{~nm}$ in horizontal and $\simeq 11 \mathrm{~nm}$ in vertical direction. Note that the resulting anisotropy of effective propagation distance and sampling along the horizontal and vertical direction can easily be implemented in the numerical Fresnel propagation. The total exposure time of the hologram was 1 second, distributed over 200 detector accumulations followed by an empty beam recording.

\section{Results}

Phase reconstructions of Deinococcus radiodurans for two different rotation angles $\theta$ are shown in Figure 3. They were obtained by averaging the complex reconstructions from 50 independent runs of the modified HIO algorithm, which stopped at an average iteration number of $N_{\text {it }} \simeq$ 2000 and showed a very small distribution of the resulting phase values with a standard deviation below $5 \cdot 10^{-4} \mathrm{rad}$
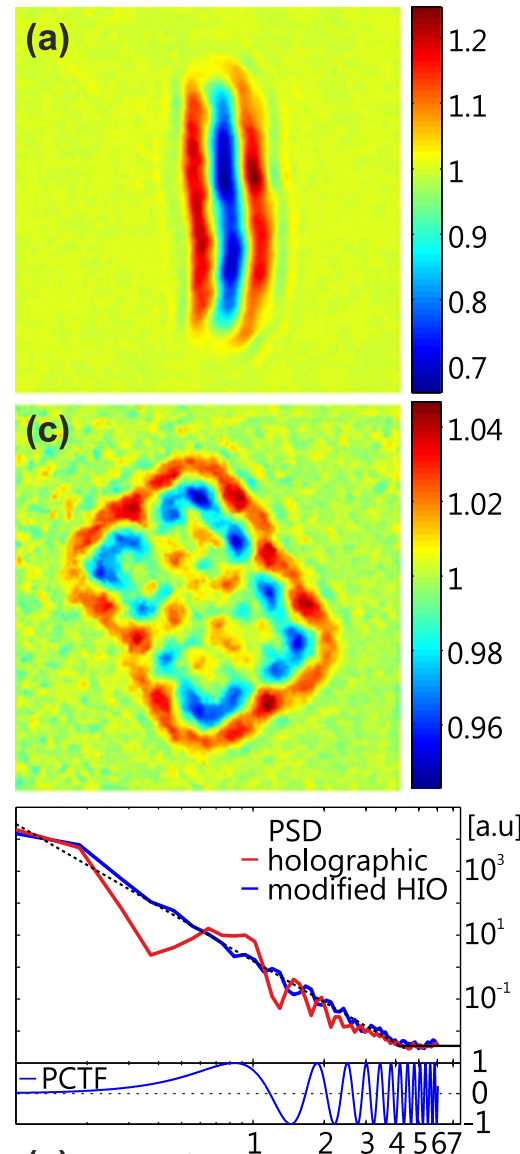

(e) Spatial frequency [periods/um]
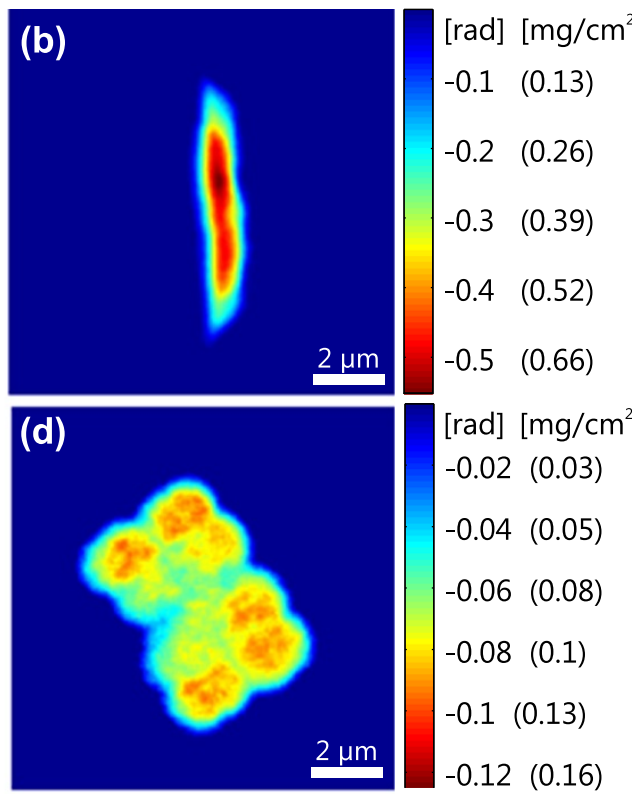

[rad] $\left[\mathrm{mg} / \mathrm{cm}^{2}\right]$

$-0.02(0.03)$

$-0.04(0.05)$

$-0.06(0.08)$

$-0.08(0.1)$

$-0.1(0.13)$

$-0.12(0.16)$

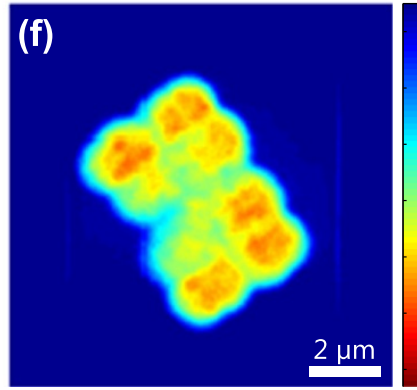

[rad] $\left[\mathrm{mg} / \mathrm{cm}^{2}\right]$

-0.03 (0.04)

-0.05 (0.07)

-0.07 (0.09)

-0.09 (0.12)

$-0.11(0.14)$

$-0.13(0.17)$

Figure $32 \mathrm{D}$ projections of $\boldsymbol{D}$. radiodurans. Holographic intensity diffracted from freeze-dried $D$. radiodurans cells at rotation angles (a) $\theta=80^{\circ}$ and (c) $\theta=0^{\circ}$, normalized by the empty WG far-field intensity distribution. (b,d) Corresponding phase reconstructions obtained with a modified $\mathrm{HIO}$ scheme as described in the main text. The coloring is scaled in rad and also mg/ $\mathrm{cm}^{2}$. (e) Angular averaged power spectral density (PSD) of the phase reconstruction shown in (d) plotted as a blue line, together with the phase-contrast transfer function (PCTF). In comparison to the PSD of a single-step holographic reconstruction, shown as a red line, the modified $\mathrm{HIO}$ scheme recovers spatial frequencies, where the PCTF is zero. A fit of

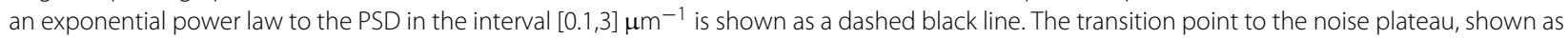
a solid black line, is at $4 \mu \mathrm{m}^{-1}$. (f) $2 \mathrm{D}$ projection of the tomographic 3D dataset of the cells, obtained by numerical evaluation of line integrals corresponding to a rotation angle $\theta=0^{\circ}$. 
in each pixel. The algorithmic feedback parameters were set to $\beta=\gamma=0.2$ and an initial guess was generated as detailed in Giewekemeyer et al. 2011. As described earlier ((Giewekemeyer et al. 2010, 2011), see also Additional file 1) the reconstructed phase distribution can be rescaled into a projected electron or, for light biological materials, mass density map (see additional colorbar scaling in Figure 3) with a density range well in agreement to what has been found before for cells of $D$. radiodurans (Giewekemeyer et al. 2010). Figure 3(e) shows the angular averaged power spectral density (PSD) of the phase reconstruction corresponding to $\theta=0^{\circ}$. Fitting the PSD to an exponential power law in the interval $[0.1,3] \mu \mathrm{m}^{-1}$, a transition to the noise plateau is found at $4 \mu \mathrm{m}^{-1}$, indicating an upper limit of the obtained (half-period) spatial resolution at $125 \mathrm{~nm} .83$ projected effective mass density maps, reconstructed from data collected at angles distributed over 162 degrees, were then used to generate a tomographic 3D effective mass density distribution of the cellular structure using standard filtered backprojection. The $2 \mathrm{D}$ projections of the tomographic 3D dataset, obtained by numerical evaluation of line integrals, shown for $\theta=0^{\circ}$ in Figure 3(f), illustrate the consistency of the independent 2D phase reconstructions. In Figure 4(a-c) different visualizations of the effective mass density distribution of the cellular structure are shown. Figure 4(d) shows a histogram of all the voxels interior of the surface defined in (b), corresponding to a cellular volume of $V=14.8 \mu^{3}$, with a total mass of $15.8 \mathrm{pg}$. All the interior voxels exhibit density values above $0.75 \mathrm{~g} / \mathrm{cm}^{3}$, however distributed in distinct spatial patterns with density maxima which can be associated with DNA rich regions. These regions exhibit densities in the range of $1.2-1.65 \mathrm{~g} / \mathrm{cm}^{3}$.
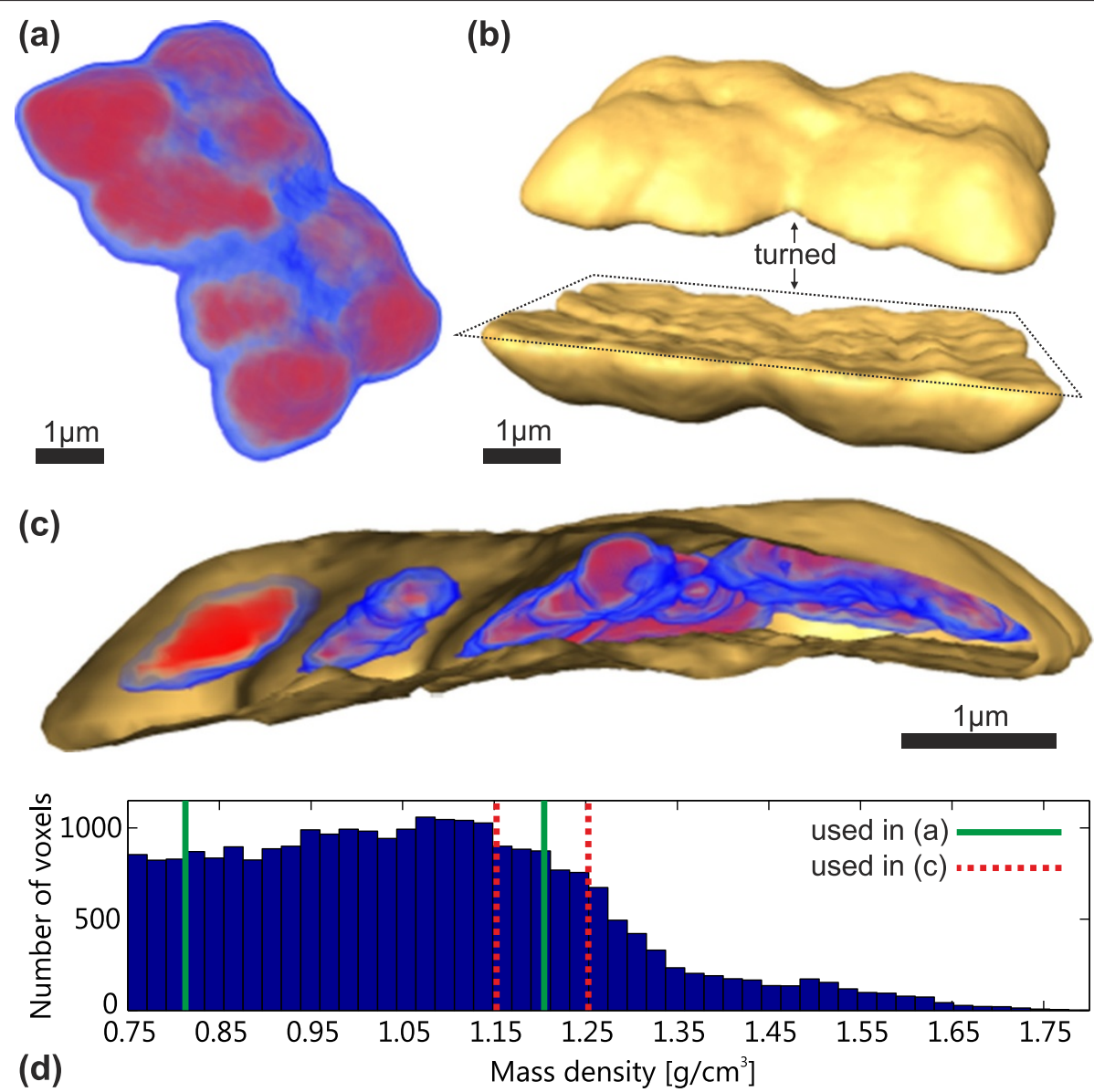

Figure 4 3D density of $D$. radiodurans. (a) Direct volume rendering of the $3 D$ effective mass density of freeze-dried $D$. radiodurans cells. The coloring indicates densities from $0.8 \mathrm{~g} / \mathrm{cm}^{3}$ (blue) to $1.2 \mathrm{~g} / \mathrm{cm}^{3}$ (red). (b) Surface rendering obtained by choosing $0.75 \mathrm{~g} / \mathrm{cm}^{3}$ as a threshold value. The cells are shown from above and turned upside-down. The presumed position of the substrate is shown as a dotted rectangle at the bottom, where the structure of the cells is nearly flat. (c) Combined direct volume rendering $\left(1.15-1.25 \mathrm{~g} / \mathrm{cm}^{3}\right)$ and surface rendering obtained by clipping the dataset with a plane. (d) Histogram of the effective mass density inside the surface shown in (b), corresponding to the volume occupied by the cells. The limits used for direct volume rendering in (a) and (c) are indicated by solid green and dashed red lines, respectively. 


\section{Conclusion}

In summary, we have used phase contrast projection tomography to compute the 3D density distribution in bacterial cells, showing characteristic density maxima which we attribute to DNA rich regions. The imaging and reconstruction method based on near-field propagation or in-line holographic recording is particularly dose efficient. Application of the method to multi-cellular organisms and tissues would allow a large field of view in all spatial dimensions at sub-cellular resolution without the need of staining or slicing. The ability to easily adapt the field of view and resolution by changing the defocus distance could be used as a zoom and advantageously combined with region-of-interest (local) tomography, which however would imply generalizations of the phase reconstruction algorithms. With further technological progress (waveguide transmission, vibrational stability), resolution can be increased up to the theoretical limit set by the extension of the quasi-point source used for illumination, which was $10 \mathrm{~nm}$ in the present case. The result of the 2D test structure, for which a waveguide with particularly high flux was available, already demonstrates that the waveguide setup is in principle well suited for imaging at a resolution below $50 \mathrm{~nm}$, a field of view in the range of several microns up to a few hundreds of microns, and one second accumulation time, paving the way for relatively fast and dose efficient nanotomography applications.

\section{Additional file}

Additional file 1: Supporting information.

\section{Competing interests}

The authors declare that they have no competing interests.

\section{Authors' contributions}

MB has been the main person carrying out the experiment and has analyzed the data. MB and TS have designed the experiment and have written the paper. MP,SK,CO,MS,SPK,RNW and TS have participated in the synchrotron experiments. SPK has contributed the $x$-ray waveguide optics, SK has contributed experimental tools, KG has contributed algorithmic tools. MP has prepared the cellular sample. All authors read and approved the final manuscript.

\section{Acknowledgements}

We thank HASYLAB for beamtime and the PETRA III team for excellent beam conditions. Funding by the DFG collaborative research center SFB 755 Nanoscale Photonic Imaging and the German Ministry of Education and Research (Grant No. 05K10MGA) is gratefully acknowledged.

\footnotetext{
Author details

${ }^{1}$ Institut für Röntgenphysik, Georg-August-Universität Göttingen, Friedrich-Hund-Platz 1, 37077 Göttingen, Germany. ${ }^{2}$ HASYLAB at DESY, Notkestr. 85, 22607 Hamburg, Germany.
}

Received: 15 July 2012 Accepted: 2 October 2012 Published: 30 November 2012

\section{References}

Cloetens P, Ludwig W, van Dyck D, et al. (1999) Holotomography: Quantitative phase tomography with micrometer resolution using hard synchrotron radiation $x$ rays. Appl Phys Lett 75: 2912

Cloetens P, Mache R, Schlenker M, et al. (2006) Quantitative phase tomography of Arabidopsis seeds reveals intercellular void network. PNAS 103: 14626

Dierolf M, Menzel A, Thibault P, et al. (2010) Ptychographic X-ray computed tomography at the nanoscale. Nature 467: 436

Eltsov E, Dubochet J (2005) Fine Structure of the Deinococcus radiodurans Nucleoid Revealed by Cryoelectron Microscopy of Vitreous Sections. J Bacteriol 187: 8047

Eltsov, E, Dubochet J (2006) Study of the Deinococcus radiodurans Nucleoid by Cryoelectron Microscopy of Vitreous Sections: Supplementary Comments. J Bacteriol 188: 6052

Eltsov, E, Dubochet, J (2006) Rebuttal: Ring-Like Nucleoids and DNA Repair in Deinococcus radiodurans. J Bacteriol 188: 6052

Fuhse C, Ollinger C, Salditt T (2006) Waveguide-Based Off-Axis Holography with Hard X Rays. Phys Rev Lett 97: 254801

Giewekemeyer K, Thibault P, Kalbfleisch S, et al. (2010) Quantitative biological imaging by ptychographic $x$-ray diffraction microscopy. PNAS 107: 529

Giewekemeyer K, Krüger SP, Kalbfleisch S, et al. (2011) X-ray propagation microscopy of biological cells using waveguides as a quasipoint source. Phys Rev A 83: 023804

Guizar-Sicairos M, Thurman ST, Fienup JR (2008) Efficient subpixel image registration algorithms. Opt Lett 33: 156-158

Gureyev TE (2003) Composite techniques for phase retrieval in the Fresnel region. Opt Commun 220: 49

Howells MR, Beetz T, Chapman HN, et al. (2009) An assessment of the resolution limitation due to radiation-damage in $\mathrm{X}$-ray diffraction microscopy. J El Spec Rel Phen 170: 4

Huang X, Nelson J, Kirz J, et al. (2009) Soft X-Ray Diffraction Microscopy of a Frozen Hydrated Yeast Cell. Phys Rev Lett 103: 198101

Kalbfleisch S, Neubauer H, Krüger SP, et al. (2011) The Gøttingen Holography Endstation of Beamline P10 at PETRA III/DESY. AIP Conf Proc 1365: 96

Krüger SP, Giewekemeyer K, Kalbfleisch S, et al. (2010) Sub-15 nm beam confinement by two crossed $\mathrm{x}$-ray waveguides. Opt Express 18: 13492

Krüger SP, Neubauer H, Bartels M, et al. (2012) Sub-10 nm beam confinement by $\mathrm{X}$-ray waveguides: design, fabrication and characterization of optical properties. J Synchrotron Rad 19: 227

Levin-Zaidman S, Englander J, Shimoni E, et al. (2003) Ringlike Structure of the Deinococcus radiodurans Genome: A Key to Radioresistance? Science 299: 254

Lima E, Wiegart L, Howells M, et al. (2009) Cryogenic X-Ray Diffraction Microscopy for Biological Samples. Phys Rev Lett 103: 198102

Mayo SC, Miller PR, Wilkins W, et al. (2002) Quantitative X-ray projection microscopy: phase-contrast and multi-spectral imaging. J Microsc 207: 79

Minsky A, Shimoni E, Englander J (2006) Rebuttal: Study of the Deinococcus radiodurans Nucleoid. J Bacteriol 188: 6059

Mokso R, Cloetens P, Ludwig W, et al. (2007) Nanoscale zoom tomography with hard $x$ rays using Kirkpatrick-Baez optics. Appl Phys Lett 90: 144104

Nishino Y, Takahashi Y, Imamoto N, et al. (2009) Three-Dimensional Visualization of a Human Chromosome Using Coherent X-Ray Diffraction. Phys Rev Lett 102: 018101

Nugent KA (2010) Coherent methods in the X-ray sciences. Adv Phys 59: 1

Paganin D (2006) Coherent X-Ray Optics. Oxford University Press, Oxford

Salditt T, Kalbfleisch S, Osterhoff M, et al. (2011) Partially coherent nano-focused $x$-ray radiation characterized by Talbot interferometry. Opt Express 19: 9656

Shapiro D, Thibault P, Beetz T, et al. (2005) Biological imaging by soft x-ray diffraction microscopy. PNAS 102: 15343

Song C, Jiang H, Mancuso A, et al. (2008) Quantitative Imaging of Single, Unstained Viruses with Coherent X Rays. Phys Rev Lett 201: 158101

Wilke RN, Priebe M, Bartels M, et al. (2012) Hard X-ray imaging of bacterial cells: nano-diffraction and ptychographic reconstruction. Opt Express 20(17): 19232-19254

Wilkins SW, Gureyev TE, Gao D, et al. (1996) Phase-contrast imaging using polychromatic hard x-rays. Nature 384: 335

doi:10.1186/2192-2853-1-10

Cite this article as: Bartels et al:: Low-dose three-dimensional hard x-ray imaging of bacterial cells. Optical Nanoscopy 2012 1:10. 